\title{
Quality in genetic counselling for presymptomatic testing - clinical guidelines for practice across the range of genetic conditions
}

\author{
Heather Skirton ${ }^{\star, 1}$, Lesley Goldsmith ${ }^{1}$, Leigh Jackson ${ }^{1}$ and Aad Tibben ${ }^{2}$
}

Presymptomatic testing (PST) is the performance of a genetic test on an asymptomatic individual at risk of a condition to determine whether the person has inherited the disease-causing mutation. Although relevant guidelines exist for specific diseases, there is no overarching protocol that can be adapted to any disorder or clinical setting in which such testing is offered. The objective of this European project was to develop a set of coherent guidelines for PST (for adult-onset monogenic conditions) for use by health professionals working in a range of disciplines, countries or contexts. To ensure the guidelines were appropriate and practice based, we organised a workshop attended by an expert group of practitioners with relevant health professional backgrounds from 11 countries. Models of service for offering PST were presented, the group then discussed different aspects of testing and the standard of care required to ensure that patients were prepared to make decisions and deal with results and consequences. After the workshop, several rounds of consultation were used with a wider group of professionals to refine the guidelines. The guidelines include general principles governing the offer of testing (eg, autonomous choice of the patient), objectives of genetic counselling in this context (eg, facilitation of decision making), logistical considerations (eg, use of trained staff) and topics to be included during counselling discussion with the patient (eg, consequences of both positive and negative outcomes). We recommend the adoption of these guidelines to provide an equitable structure for those seeking PST in any country.

European Journal of Human Genetics (2013) 21, 256-260; doi:10.1038/ejhg.2012.174; published online 15 August 2012

Keywords: presymptomatic testing; predictive testing; guidelines; recommendations

\section{INTRODUCTION}

Presymptomatic testing (PST) is defined as the performance of a genetic test on an asymptomatic individual to determine whether or not the person has inherited the disease-causing mutation. ${ }^{1}$ The terms 'predictive testing' or 'susceptibility testing' may sometimes be used in the same context, but for the purposes of this paper we will use PST.

As recombinant DNA techniques enabled testing of samples through linkage analysis in the 1980's, PST for a range of genetic conditions has been possible. For example, testing for Huntington's disease had been possible via linkage analysis since before $1983,{ }^{2}$ although some patients preferred to have a more accurate direct test, ${ }^{3}$ which became available after the mutations responsible for this and other neurodegenerative disorders were identified. ${ }^{4}$ Later, as understanding of familial cancer syndromes increased, PST was offered to individuals at increased risk of breast and ovarian cancer, $^{5}$ Lynch syndrome (previously termed hereditary nonpolyposis cancer) ${ }^{6}$ and familial adenomatous polyposis. ${ }^{7}$

The information now available about specific disease-causing mutations has enabled PST to be offered for a much wider range of disorders and it may now be provided by health professionals outside of specialist genetic centres. For example, testing for the mutations that cause hypertrophic cardiomyopathy, ${ }^{8}$ or maturity onset diabetes of the young ${ }^{9}$ may be offered in cardiac and diabetes clinics, respectively. It has been noted that the philosophical and practical approach to offering testing may differ between specialist genetic centres and other health services. ${ }^{10}$ It is therefore important that guidance is available on the requirements for PST for adult-onset conditions to ensure patient choice and safety.

\section{BACKGROUND LITERATURE}

Formal guidelines for offering PST in a clinical context were developed in 1989 and updated in 1994 by the World Federation of Neurology Working Group on Huntington's disease and International Huntington's Association, ${ }^{11}$ and a second update has been published recently. ${ }^{12}$ The guidelines are primarily for use with Huntington's disease but can also be applied for other neurological conditions (eg, Alzheimer disease, frontotemporal disease, spino-cerebellar ataxia). The tenets of the document are that: the decision to take a test should be voluntary, without coercion from any other person, informed consent should be sought, the individual should have access to counselling of the highest quality and testing should be offered within specialist genetic units. Specific and highly detailed recommendations are made about the information to be given during pre-test counselling including information on the test procedure, alternatives and consequences. Post-test counselling sessions should be planned before the result is given and include the need for the counsellor to initiate contact after 1 month if it has not been made by the patient. Other authors ${ }^{13}$ refer to these

${ }^{1}$ Faculty of Health, Education and Society, Plymouth, University, Taunton, UK; ${ }^{2}$ Centre for Human and Clinical Genetics, Leiden University, Medical Centre, Leiden, The Netherlands

*Correspondence: Professor H Skirton, Faculty of Health, Education and Society, Plymouth University, Wellington Road, Taunton TA1 5YD, UK. Tel: +1 823366911 ; Fax: +1 823 366901; E-mail: heather.skirton@plymouth.ac.uk

Received 26 April 2012; revised 25 June 2012; accepted 6 July 2012; published online 15 August 2012 
prescriptive guidelines for use in PST conditions other than Huntington's disease, for example Panegyres et al ${ }^{13}$ refer to these in the context of testing for familial Alzheimer disease. The Huntington's disease model is also consistent with the guidelines adopted for testing for non-neurodegenerative conditions, such as hereditary forms of cancer ${ }^{14,15}$ and cardiogenetic conditions. ${ }^{16,17}$

Dufrasne et $a^{18}$ described the use of the Huntington's protocol in detail after 15 years of experience in using it in Canada. In summary, the counselling was offered by a multi-disciplinary team comprising medical geneticists, genetic counsellors and psychologists. Before faceto-face contact the patients discussed their wish for a test by telephone with a psychologist. Following that call, three face-to-face contacts were made, the first $(2 \mathrm{~h})$ was with a psychologist to assess psychological readiness and needs of the patient. In the second $(1 \mathrm{~h})$, the patient was seen by a physician and the psychologist, to obtain medical history and confirm the diagnosis of Huntington's disease in the family. The third session $(2 \mathrm{~h})$ was conducted with a genetic counsellor. If the patient proceeded with testing, the results were disclosed at a fourth session and a follow-up consultation was arranged by telephone 1 week after the results were given. Of an initial 135 patients who requested testing, 40 withdrew during the pre-test counselling process. Of these, 18 withdrew after the initial telephone call with the psychologist and the authors attribute this to last minute self-selection, possibly by those who are highly ambivalent about their desire to be tested.

Brain et $a l^{19}$ gathered data on the protocols used by 16 genetics centres offering PST for Lynch syndrome. All centres offered a minimum of one pre-test counselling session, although the range was 1-3 sessions. Where more than one session was offered, the time period between them was four to 8 weeks. Staff of four of the centres reported, however, that more than one session was often unnecessary and that they had reduced the number of pre-test sessions to one or were planning to do so. A particular point was made that the situation differed from that of Huntington's disease, because families seemed more 'matter of fact'19 (p200) about the testing and did not want the testing procedure to be needlessly prolonged.

In a study of 271 adults tested for a Lynch syndrome mutation, Aktan-Collan et al ${ }^{10}$ noted that participants felt the strongest need for psychological support at the time of disclosure of the result, suggesting that having a personal friend or family member present might be useful in this regard. However, although bringing a support person to the results session had been recommended, only $30 \%$ of those in the study had done this. This highlights the conundrum between enabling choice and setting firm criteria for testing. In that study, pre-test counselling had included topics such as the actual mutation, methods of screening for tumours, benefits and disadvantages of testing and emotional responses to testing. At the post-test session, results were given as well as further reinforcement of information about screening for mutation positive patients and reminder of the population risk of colon cancer for those who were mutation negative.

Although sets of guidelines for offering PST have been developed, these relate to specific disorders or groups of disorders. In the current era of genetic and genomic health care, PST is available for increasing numbers of diseases, and is provided by health professionals working outside the genetic specialist clinics. ${ }^{8,9,20}$ There is no overarching protocol or guidance that can be adapted to any disorder or clinical setting in which PST is offered. The objective of the EuroGentest2 project is to ensure that genetic testing is provided equitably and safely to patients in Europe. One of the deliverables for the EuroGentest2 project is therefore to develop a set of coherent guidelines for PST that can be used by health professionals working in a range of countries or contexts to ensure that patients are appropriately prepared for such testing and the consequences of the test.

\section{METHODS}

The leaders of the EuroGentest2 project work package are experienced clinical health professionals in the field of genetics. However, we felt it was important to utilise the experience of a wide range of practitioners from across Europe and so we used an expert group to develop guidelines for counselling when offering PST for adult-onset monogenic conditions.

\section{Participants}

Experienced practitioners were invited to attend a workshop to discuss, debate and formulate the key requirements for PST counselling. Practitioners with relevant professional backgrounds from 11 countries attended (Table 1). Of these, a core group of those who were highly experienced in PST counselling focussed on development of the guidelines. However, a wider group of practitioners contributed through discussion of the outputs.

\section{Process}

All participants were made aware of the focus of the workshop in the invitation to attend. A number of relevant publications ${ }^{17,21-23}$ were provided to each participant several weeks before the workshop and they were asked to read these before arrival to help them prepare for the discussions.

During the first session of the workshop, presentations were made by a medical oncologist offering PST in the context of familial cancer syndromes, a medical cardiologist providing PST to those at risk of cardiac genetic conditions and a nurse consultant who pioneered PST for monogenic forms of diabetes and set up a network to train professionals to offer such testing nationally. All three presented models of service for offering PST.

The entire group then discussed the terms of reference for the guidelines before splitting to discuss different aspects of service. The core group worked specifically on quality of genetic counselling to support PST. Twice during the workshop the groups reconvened for discussion and feedback from the wider group on the developing guidelines.

There will be occasions when PST may be offered to or sought by minors, ${ }^{24,25}$ but the expert group decided not to specifically address this

\section{Table 1 Profession and country of workshop participants}

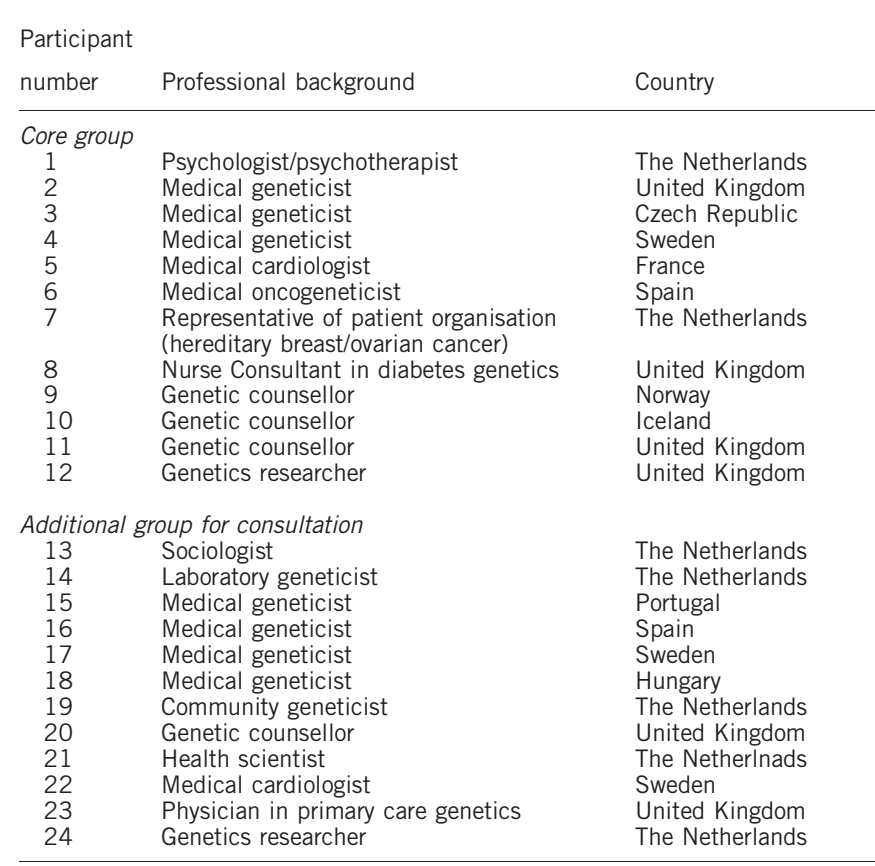


issue as other guidance exists, to which practitioners should refer. $^{26,27}$ Following the workshop, the guidelines were circulated to all members for further comments and minor revisions were made.

\section{RESULTS}

The objective of counselling for PST is to enable the patient to make an informed and autonomous choice, to adjust to the results of the test (should the test be performed) in the context of his or her own life and utilise the results for health care management. For the purposes of these guidelines, PST is defined as testing of an asymptomatic adult for a mutation for a specific monogenic disorder. In these cases, where possible it is expected that the laboratory staff will aim to use a method that limits results to the clinical question being answered. However, if targeted mutation testing is not feasible, the possibility of other findings must be discussed with the patient before the test is performed and a disclosure strategy for any unexpected (incidental) findings agreed with the patient (for example, if whole genome sequencing is used, it would be possible to identify a BRCA1 mutation in an individual being tested for a neurodegenerative condition).

The principles provide a roadmap to autonomous choice. These include:

(1) establishment of a respectful relationship between counsellor and patient, in which the patient's expectations, values and agenda have priority

(2) employment of appropriately trained and skilled people to provide the counselling (see relevant documents)

(3) provision of 'adequate' information (see content below) based on patient's needs and expectations, to include:

- disease-specific information

- psychological impact of testing

- social implications of testing

- legal implications of testing

(4) enabling the patient to weigh the consequences and benefits in the context of his/her life (with relation to thoughts, values, beliefs, feelings)

(5) Counselling that leads to autonomous choice

(6) Counselling undertaken in the context of a professional code of conduct.

\section{OBJECTIVES}

The objectives of making PST available to unaffected (usually adult) family members at risk are to:

(1) facilitate the patient in making his or her own decision about testing by giving information and supporting exploration of the context and consequences of the decision they make

(2) provide the opportunity for self-management re:

- health care

- personal wellbeing and adjustment

- reproduction

- family communication.

\section{LOGISTICS}

It is important that an individualised approach is taken regarding the number and timing of counselling sessions, based on stage of maturation of patient rather than the particular disease. In general, a verbal (and if appropriate, written) summary of the issues discussed in counselling should be made at the end of each session and a letter with relevant information about the disease and risk transference patterns for the family should also be provided to facilitate family disclosure.

The counsellor is responsible for making appropriate referrals and providing a comprehensive handover to other health professionals, including relevant psychological social or legal issues. The plan for coordination of care should be agreed with other health professionals.

It is assumed that health professionals providing PST will be appropriately trained and possess the relevant competences. ${ }^{28}$ It is particularly important in the context of PST that health professionals recognise their own limitations and are able to make referrals to other health professionals (such as psychologists or psychotherapists) if required to support assessment of the patient or to provide the patient with psychological support.

(1) Pre-test counselling

Pre-test counselling is preferably done via a face-to-face conversation. The diagnosis, family history and risk to the individual will provide a foundation for the discussion. It should also include:

- information about the test and testing process, including likelihood of definitive results and what will happen to the remainder of the DNA sample after the test is performed

- the scope of the test and what it will show (and what it will not)

- the patient's perspective and wishes for and expectations of a test

- information about waiting time for test

- a clear indication of time between the sample being taken and disclosure of results

- opportunity to have a support person present during the pretest, disclosure session and post-test sessions

- consent for testing and documentation of the decision

- an agreed method of conveying results (e-mail, phone, face-toface, letter)

- the plan for follow-up after the results disclosure

- plans for disclosure to other family members

- information on support groups, surveillance and/or treatment

- specific issues of relevance to the particular disease

- implications for offspring (where applicable, to include discussion of reproductive choices)

- meaning of the possible results (technical and personal significance)

- relevance of the results to other relatives who may not have requested testing

- discussion of options available regarding screening and/or treatment and which of these could be used regardless of whether PST is performed

- discussion of psychological issues including coping with disclosure of test results, removal of uncertainty, residual uncertainty (where appropriate) and dealing with changed genetic status

- discussion of social issues including impact of testing (or not) on employment, insurance, personal and family relationships, privacy and confidentiality. This should include the implications of testing and/or results for the wider family, including partner or spouse

- the availability of follow-up and support after testing

- invitation for questions and offering of additional information as required.

The order of priority for discussion of topics will vary depending on condition or family situation. 
(2) Post-test disclosure and counselling

Post-test counselling should include:

- appropriate disclosure of results, as previously agreed with patient

- discussion of plan for future contact

- re-iteration of information on support groups, surveillance, treatment, referrals to other practitioners or services and, where appropriate, participation in research projects

- the availability of follow-up and support.

In addition, some of the topics covered in the pre-test session may need to be revisited during the post-test counselling sessions; for example, the implications for the patient and relatives and discussion of the psychological impact of changed genetic status.

Note that the patient may prefer to receive results at a face-to-face meeting, by telephone, by letter or by electronic communication. This should be agreed during pre-test counselling. If the results are not delivered in person (face-to-face or by telephone), counselling should be offered. This should be offered regardless of whether the patient has a positive or negative mutation result, as it has been shown that patients who are mutation negative can also struggle with the psychological impact of the results. ${ }^{29}$

\section{DISCUSSION}

PST has become available for a great variety of disorders. Where initially PST was offered mainly in the context of untreatable diseases (for the sake of pre-warning of the condition and psychological preparation for the future), it is now also used in situations where there is a greater perceived medical benefit from testing (eg, for conditions such as hypertrophic cardiomyopathy, ${ }^{8}$ maturity onset diabetes of the young ${ }^{9}$ ). It is important in all situations that individuals still make individual choices based on their own values, beliefs and preferences. As the study by Dufrasne et al ${ }^{12}$ indicated, even those who actively request PST may be ambivalent about testing and appropriate counselling must be available to enable them to make informed choices. The overarching set of recommendations presented in this paper are consistent with those developed for specific monogenic neurodegenerative conditions ${ }^{10,11}$ such as Huntington's or Alzheimer disease, hereditary forms of cancer and hereditary forms of hereditary cardiac conditions, with the emphasis on voluntary action on the part of the patient and informed choice.

We emphasise that where appropriate disease-specific guidelines exist, they should still be used. However, there are a great many conditions for which PST is or will be available for which no such protocols have been prepared. The objectives, counselling content and logistical requirements have been documented here to provide a template for PST in a range of professionals and clinical contexts.

In developing these guidelines, the expert group deliberately focussed on the principles and objectives of PST rather than making prescriptions in terms of numbers of sessions of counselling. This enables practitioners to use their own professional judgement to individualise the process, as shown to be necessary by Brain et al ${ }^{13}$ The opportunity for support during and after disclosure is emphasised as this has been identified as an area of need by patients undergoing PST. ${ }^{14}$

The guidelines emphasise the involvement of appropriately trained professionals in offering PST. Although this will often mean genetic specialists such as medical geneticists or genetic counsellors, increasingly specialists in other secondary care settings will be providing PST. Their particular specialist knowledge is an obvious advantage in discussing the potential natural history of the disease and preventive measures or management. However, the experience of practitioners working in other fields may differ in some respects from those working in genetics. For example, genetics specialists are constantly required to use an approach that involves consideration of the immediate and wider family ${ }^{30}$ and because of the history of genetic counselling may be more practised in using a nondirective approach. $^{31}$ It is therefore essential that all health professionals providing PST, whether in clinical genetics or other disciplines, should have appropriate education and training in the requisite skills to ensure patients are able to exercise freedom of choice and genuinely consider the implications of testing for themselves and their families. To this end, a set of core competences in genetic health care and relevant learning outcomes have been written for professionals working in a range of disciplines in primary, secondary and tertiary settings. ${ }^{28}$ Of particular importance in the context of PST are the competences related to appropriate counselling skills and ability to offer psychological support to the patient. In order to attain these skills, specific education in counselling skills is required, as well as ongoing counselling supervision for professionals undertaking these tasks.

\section{CONCLUSIONS}

These recommendations have been produced by specialists in Europe for use in any setting where PST for genetic disorders caused by a single gene mutation is offered. The objective was to ensure testing was provided in a way that facilitated patient choice while maintaining patient safety. We recommend their adoption to provide an equitable structure for those seeking testing in any European country.

\section{CONFLICT OF INTEREST}

The authors declare no conflict of interest.

\section{ACKNOWLEDGEMENTS}

This study was funded under the EuroGentest Co-ordination Action 2011 — EU Contract no.: HEALTH-F4-2010-2614692 project. We acknowledge the support of Tessel Rigter in helping to organise the workshop. The workshop participants who contributed greatly to the guidelines were: Inga Bjornevoll, Ignacio Blanco, Angus Clarke, Vaclava Curtisova, Lidewij Henneman, Anke Liebbrandt, Christine Patch, Nadeeem Qureshi, Carolyn Rooryck-Thambo, Maggie Shepherd, Maria Soller, Vigdis Stefansdottir. We also thank the following persons for their additional comments: Johan Brandt, Martina Cornel, Helena Kaariainen, Ulf Kristoffersson, Bela Melegh,Marcel Nelen, Feliciano Ramos, Jorge Sequeiros, Carla van El.

1 Paneque M, Sequeiros J, Skirton H: Quality assessment of genetic counselling process in the context of presymptomatic testing for late-onset disorders: athematic analysis of three review articles. Genet Test Mol Bioma 2012; 16: 36-45.

2 Gusella JF, Wexler NS, Conneally PM et al: A polymorphic DNA marker genetically linked to Huntington's disease. Nature 1983; 306: 234-238.

3 Beilby J, Chin CY, Porter I, Walpole IR, Goldblatt J: Improving diagnosis of Huntington's disease by analysis of an intragenic trinucleotide repeat expansion. Med J Aust 1994; 161: 356-360.

$4 \mathrm{La}$ Spada AR, Paulson HL, Fischbeck KH: Trinucleotide repeat expansion in neurological disease. Ann Neurol 1994; 36: 814-822.

5 Weber BL, Abel KJ, Brody LC et al: Familial breast cancer. Approaching the isolation of a susceptibility gene. Cancer 1994; 74: 1013-1020.

6 Stanley AJ, Gaff CL, Aittomäki AK, Fabre LC, Macrae St FA, John J: Value of predictive genetic testing in management of hereditary non-polyposis colorectal cancer (HNPCC). Med J Aust 2000; 172: 313-316.

7 Karner-Hanusch J, Wolf B, Zehetmayer M, Wrba F, Roth E, Mannhalter C: Screening by genomic linkage studies and mutation analysis of hereditary adenomatous polyposis coli: usefulness for clinical practice. World J Surg 1996; 20: 578-583.

8 Michels M, Hoedemaekers YM, Kofflard MJ et al: Familial screening and genetic counselling in hypertrophic cardiomyopathy: the Rotterdam experience. Neth Heart $J$ 2007: 15: 184-190.

9 Shepherd M, Ellis I, Ahmad AM et al: Predictive genetic testing in maturityonset diabetes of the young (MODY). Diabetic Med 2001; 18: 417-421. 
10 Aktan-Collan K, Mecklin JP, de la Chapelle A, Peltomäki P, Uutela A, Kääriäinen $H$ Evaluation of a counselling protocol for predictive genetic testing for hereditary non-polyposis colorectal cancer. J Med Genet 2000; 37: 108-113.

11 International Huntington Association (IHA) and the World Federation of Neurology (WFN) Research Group on Huntington's Chorea: Guidelines For The Molecular Genetics Predictive Test In HD 1994.

12 MacLeod RTA, Frontali M, Evers-Kiebooms G, Jones A, Martinez-Descales A, Roos R and editorial committee and working group. 'Genetic testing and Counselling' of the European Huntington Disease Network, Recommendations for the predictive genetic test in Huntington's disease. Clinical Genetics 2012; e-pub ahead of print 29 July 2012; doi: 10.1111/j.1399-0004.2012.01900.x.

13 Panegyres PK, Goldblatt J, Walpole I, Connor C, Liebeck T, Harrop K: Genetic testing for Alzheimer's disease. Med J Aust 2000; 172: 339-343.

14 Marshall M, Solomon S: Hereditary breast-ovarian cancer: clinical findings and medical management. Plast Surg Nurs 2007; 27: 124-127.

15 Nippert I, Schlegelberger B: Women's experiences of undergoing BRCA1 and BRCA2 testing: organisation of the German Hereditary Breast and Ovarian Cance Consortium Survey and Preliminary Data from Münster. Commun Genet 2003; 6 249-258.

16 Gollob MH, Blier L, Brugada R et al: Recommendations for the use of genetic testing in the clinical evaluation of inherited cardiac arrhythmias associated with sudden cardiac death: Canadian Cardiovascular Society/Canadian Heart Rhythm Society joint position paper. Can J Cardiol 2011; 27: 232-245.

17 Christiaans I, van Langen IM, Birnie E, Bonsel GJ, Wilde AAM, Smets EMA: Genetic counseling and cardiac care in predictively tested hypertrophic cardiomyopathy mutation carriers: the patients' perspective. Am J Med Genet A 2009; 149A: 1444-1451.

18 Dufrasne S, Roy M, Galvez M, Rosenblatt DS: Experience over fifteen years with a protocol for predictive testing for Huntington disease. Mol Genet Metab 2011; 102 494-504.

19 Brain K, Soldan J, Sampson J, Gray J: Genetic counselling protocols for hereditary non-polyposis colorectal cancer: a survey of UK regional genetics centres. Clin Genet 2003; 63: 198-204.
20 Calzone KA, Stopfer J, Blackwood A, Weber BL: Establishing a cancer risk evaluation program. Cancer Pract 1997; 5: 228-233.

21 Charron $\mathrm{P}$, Héron D, Gargiulo $\mathrm{M}$ et al: Genetic testing and genetic counselling in hypertrophic cardiomyopathy: the French experience. J Med Genet 2002; 39: 741-746.

22 Boenink M: Unambiguous test results or individual independence? The role of clients and families in predictive BRCA-testing in the Netherlands compared to the USA. Soc Sci Med 2011; 72: 1793-1801.

23 Elwyn G, Gray J, Clarke A: Shared decision making and non-directiveness in genetic counselling. J Med Genet 2000; 37: 135-138.

24 Borry P, Goffin T, Nys H, Dierickx K: Predictive genetic testing in minors for adult-onset genetic diseases. Mt Sinai J Med 2008; 75: 287-296.

25 Borry P, Goffin T, Nys H, Dierickx K: Attitudes regarding carrier testing in incompetent children: a survey of European clinical geneticists. Eur J Hum Genet 2007; 15: 1211-1217.

26 European Society of Human Genetics. Genetic testing in asymptomatic minors: recommendations of the European Society of Human Genetics. Eur J Hum Genet 2009; 17: 720-721.

27 British Society for Human Genetics, Report on the Genetic Testing of Children 2010 http://www.clingensoc.org/Docs/GTOC\%20BSHG\%202010.pdf 2010.

28 Skirton H, Lewis C, Kent A, Coviello DA: Members of Eurogentest U, Committee EE: genetic education and the challenge of genomic medicine:development of core competences to support preparation of health professionals in Europe. Eur J Hum Genet 2010; 18: 972-977.

29 Gargiulo M, Lejeune S, Tanguy ML et al: Long-term outcome of presymptomatic testing in Huntington disease. Euro J Hum Genet 2009; 17: 165-171.

30 Wright C, Kerzin-Storrar L, Williamson PR et al: Comparison of genetic services with and without genetic registers: knowledge, adjustment, and attitudes about genetic counselling among probands referred to three genetic clinics. J Med Genet 2002; 39: e84-e84.

31 Kessler S: Psychological aspects of genetic counseling XIV. Nondirectiveness and counseling skills. Genet Test 2001; 5: 187-191. 